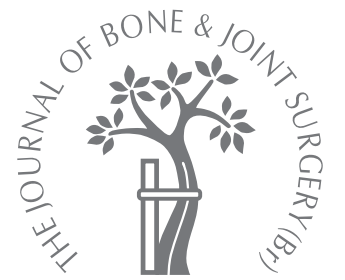

A. Rajpura,

B. V. Somanchi,

L. T. S. W. Muir

From Hope Hospital, Salford, England

\title{
The effect of tourniquet padding on the efficiency of tourniquets of the upper limb
}

\begin{abstract}
We report the effect of padding on the efficiency of the pneumatic tourniquet for the upper limb. Varying thicknesses of two commercially-available types of orthopaedic padding (Cellona and Velband) were applied to the arms of 20 volunteers, with three pressure transducers placed directly beneath the padding. A tourniquet was positioned over the padding and inflated to $220 \mathrm{mmHg}$.

Significant reductions in the transmitted pressure were recorded from the transducers with both padding materials. With eight layers of padding, reductions in pressure of $\mathbf{1 3} \%$ $(1 \%$ to $26 \%)$ and $18 \%$ (7\% to $35 \%)$ were seen with Cellona and Velband, respectively. The reduction in pressure with Velband padding correlated with increasing arm circumference (Pearson's correlation coefficient $0.711, \mathrm{p}<0.001$ ).

Studies to date have examined how arm circumference affects the required tourniquet inflation pressure. Our study is the first to investigate the effect of the padding and the findings suggest that using more than two layers results in a significant reduction in the transmitted pressure.
\end{abstract}

The tourniquet is almost indispensable in orthopaedic practice. The ability to obtain an operative field with little or no bleeding helps to provide a clear view and reduces blood loss. The modern pneumatic tourniquet was originally devised by Cushing in $1904^{1}$ and with little change remains in contemporary use for most surgery on the upper and lower limbs. ${ }^{2}$

In order to ensure its safe use Bruner published his 'ten rules' in $1951^{3}$ which were subsequently modified by Braithwaite and Klenerman ${ }^{4}$ and Kutty and McElwain. ${ }^{5}$ One rule was to use padding beneath the tourniquet in the form of at least two layers of orthopaedic wool to reduce the chances of trauma to the skin.

The influence of the circumference of the arm and thickness of the subcutaneous layer of fat on the minimum tourniquet pressures required to maintain a bloodless field have been reported. ${ }^{6,7}$ However, the effect of the padding on the efficiency of the tourniquet has not been investigated. Anecdotal evidence suggests that excessive padding reduces the pressure exerted and can lead to failure of the tourniquet.

We have therefore studied the effect of the tourniquet padding on the amount of pressure transmitted to the underlying limb.

\section{Patients and Methods}

We recruited 20 healthy adult volunteers for the study, ten male and ten female, and investigated the difference between two types of commonly used orthopaedic wool, Cellona (Lohmann \& Rauscher, s.r.l. Neuwied, Germany) and Velband (BSN Medical Ltd, Nelson, United Kingdom). A standard 460 mm $\times 100 \mathrm{~mm}$ tourniquet for the upper limb (Anetic Aid Ltd, Guiseley, United Kingdom) with a calibrated APT Mk3 Tourniquet Control Unit (Anetic Aid Ltd) was used for all examinations. A proprietary three-channel pressure measurement device (Medical Physics Dept, Hope Hospital, Salford, United Kingdom) was used and was calibrated against a Druck DPI 800 Pressure Calibrator (GE Druck, Leicester, United Kingdom).

The arm circumference for each subject was measured with a tape measure at the mid-point between the olecranon and acromion and three pressure transducers were then attached at this level, one anteriorly, one posteriorly and one medially. The tourniquet was then applied in a standard manner without any padding ensuring that the minimum overlap specified by the manufacturer was obtained. The tourniquet was centred over the midpoint of the arm. It was then inflated until $220 \mathrm{mmHg}$ was registered on the control unit. This is the standard 
Table I. Results for measured pressures in $\mathrm{mmHg}$ (mean, range, percentage compared with 0 layer group) with differing levels of padding

\begin{tabular}{llll}
\hline $\begin{array}{l}\text { Amount of padding } \\
\text { (layers) }\end{array}$ & Number Cellona & Velband \\
\hline 0 & 20 & $218.7(205.0$ to 234.7) & $218.7(205.0$ to 234.7) \\
2 & 18 & $211.1(198.3$ to 225.0) & $207.8(192.7$ to 222.0) \\
& & 96.5 & 95.0 \\
4 & 20 & $206.8(172.0$ to 222.3) & $194.1(161.3$ to 217.7) \\
& & 94.6 & 88.8 \\
8 & 20 & $189.7(158.7$ to 214.0) & $179.3(142.7$ to 211.7) \\
& & 86.7 & 82.0
\end{tabular}

inflation pressure for surgery on the upper limb in our department. The pressure transmitted to the arm was measured on the three-channel monitor. After deflation of the tourniquet two layers of Cellona padding were then carefully wrapped around the arm over the pressure monitors ensuring that there were no creases. The tourniquet was reinflated to $220 \mathrm{mmHg}$ and new pressure measurements were obtained. It was estimated that each layer of padding was approximately $1 \mathrm{~mm}$ thick and would therefore increase the diameter of the arm by $4 \mathrm{~mm}$ if it were accepted that the cross-section of the arm approximated to a circle. In these circumstances the overlap of the tourniquet was reduced by approximately $12 \mathrm{~mm}(4 \mathrm{~mm} \times \pi)$, an application to ensure that the resting pressure exerted by the tourniquet was the same for each measurement. This was confirmed by recording the pre-inflation pressures, which were generally approximately $10 \mathrm{mmHg}$. The entire procedure was then repeated with four and eight layers of padding. The overlap was adjusted based upon the equation previously mentioned. The same sequence was then undertaken using Velband padding.

Statistical analysis. The mean of the three transducer readings was calculated, and the differences in the pressures measured for the various amounts of padding applied were analysed by single-factor repeated-measure multivariate analysis of variance (ANOVA). Differences in the pressure reduction between the two types of padding were analysed using paired sample $t$-tests. The statistical program SPSS version 13 (SPSS Inc., Chicago, Illinois) was used. Statistical significance was set at a $\mathrm{p} \leq 0.05$.

\section{Results}

The addition of padding beneath the tourniquet resulted in a reduction in the mean pressure recorded by the transducers applied to the skin. The means and ranges are presented in Table I. With eight layers of padding, mean reductions in pressure of $13 \%$ (1\% to $26 \%)$ and $18 \%(7 \%$ to $35 \%$ ) were seen with Cellona and Velband, respectively. With four layers, mean reductions of $5 \%(1 \%$ to $20 \%)$ and $11 \%(2 \%$ to $27 \%)$ were seen with Cellona and Velband, respectively. Single-factor repeated-measure multivariate ANOVA showed a significant effect for layers of padding with both Cellona $(\mathrm{p}<0.0005$, and Velband $(\mathrm{p}<0.0005)$

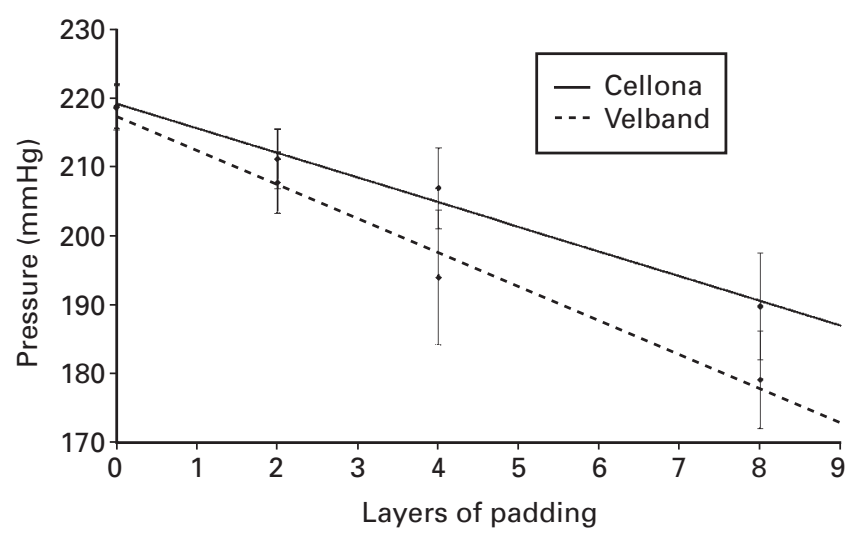

Fig. 1

Graph showing mean pressure and $95 \%$ confidence intervals $(\mathrm{Cl})$ for CelIona and Velband padding against the number of layers applied.

(Fig. 1). Paired sample $t$-tests showed that Velband padding caused a statistically significant greater reduction in pressure than Cellona padding with both four layers $(\mathrm{p}=0.01)$ and eight layers $(\mathrm{p}=0.01)$, but not with two layers $(\mathrm{p}>$ 0.1 ). We also examined the relationship between the circumference of the arm and the mean reduction in pressure due to padding (mean pressure with no padding minus mean pressure with padding) (Fig. 2). The Cellona group showed little evidence of a trend among the subjects (Pearson's correlation coefficient $0.271, p=0.276$ ), but the Velband group had a positive correlation between the observed pressure decrease due to padding and arm circumference when the two outlying values were discounted (Pearson's correlation coefficient $0.711, \mathrm{p}<0.001$ ). A significant positive correlation was also seen in the four-layer Velband group (Pearson's correlation coefficient 0.617, p = 0.006 ), but in no other group. If the mean arm circumference of $28.3 \mathrm{~cm}$ is used to separate the subjects into two groups, the mean pressure reduction with eight layers of Velband was $45.4 \mathrm{mmHg}(20 \%)$ for the $>28.3 \mathrm{~cm}$ group and $34.6 \mathrm{mmHg}(16 \%)$ for the $<28.3 \mathrm{~cm}$ group. With four layers of Velband the mean pressure reduction was $35.1 \mathrm{mmHg}(16 \%)$ for the $>28.3 \mathrm{~cm}$ group and $15.2 \mathrm{mmHg}$ $(7 \%)$ for the $<28.3 \mathrm{~cm}$ group.

\section{Discussion}

Studies concerning pneumatic tourniquets have focused on how factors such as the circumference of the arm and systolic blood pressure affect the minimum required inflation pressures. Van Roekel and Thurston ${ }^{7}$ suggested that an inflation pressure of $200 \mathrm{mmHg}$ was required for an average-sized normotensive subject. Cadaver studies have shown that the transmitted pressure within the limb is inversely proportional to the circumference of the arm. ${ }^{8}$ However, there have been no studies on the influence of the tourniquet padding on the transmitted pressure. 


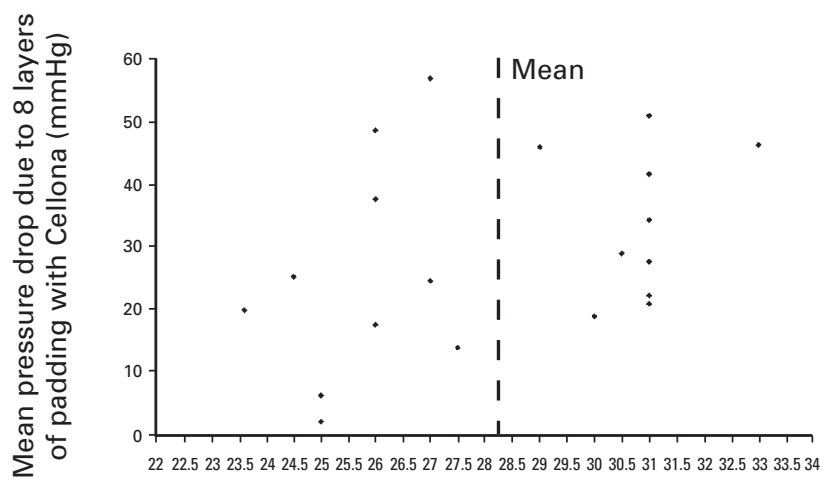

Arm circumference $(\mathrm{cm})$

Fig. 2a

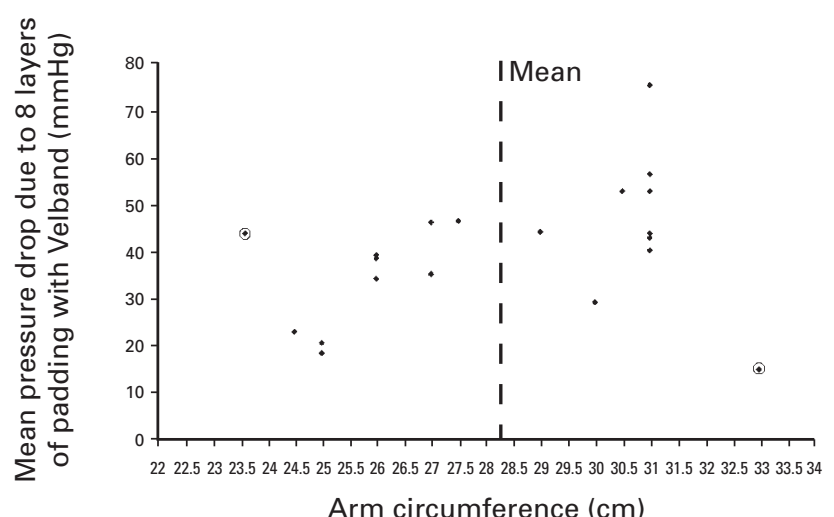

Fig. $2 b$

Scatterplot of the mean pressure reduction (mean pressure without pad ding minus mean pressure with padding) vs arm circumference for each subject with eight layers of a) Cellona and b) Velband padding. The verti cal line shows the mean arm circumference of $28.3 \mathrm{~cm}$.

Our study has shown that if a tourniquet is applied over eight layers of Velband padding, the pressure exerted on the arm may be reduced by a mean of $18 \%$. The reduction in pressure was less marked with Cellona padding, but was still a mean of $13 \%$ with eight layers of padding. In both of these situations the effectiveness of the tourniquet is likely to be affected. Padding with eight layers seemed to be excessive, but personal observation has shown that theatre staff often use the entire roll of padding which approximates to eight layers. Even with only four layers of padding a decrease of $5 \%$ was seen with Cellona and of $11 \%$ with Velband. The difference between the two materials is prob- ably due to the three-layer structure of Velband, rendering it less compressible and transmitting less pressure than Cellona which has a monolayer structure. ${ }^{9}$

The effect of padding may also be exaggerated with increasing circumference of the arm. A positive correlation between the pressure reduction and arm circumference was noted in the four- and eight-layer Velband group. Significant correlations were not found with Cellona padding, but this may have been due to the small number of subjects in the study. The reduction in pressure exerted by the tourniquet may have been due to a reduction in the amount of overlap between the ends of the tourniquet as the circumference of the arm increased, possibly reducing the efficiency of the device.

In conclusion, our data show that excessive tourniquet padding may reduce the efficiency of the tourniquet and this effect is amplified with increasing arm circumference. We also demonstrated that the reduction in pressure is greater with Velband than with Cellona padding. From these data we suggest a further modification to Bruner's ten rules in that tourniquet padding should not exceed two layers of padding in order to avoid a significant reduction in the transmitted pressure beneath the tourniquet.

\section{Supplementary Material}

$\because$ A further opinion by Professor Leslie Klenerman is available with the electronic version of this article on our website at www.jbjs.org.uk

The authors wish to thank the Department of Medical Physics, Hope Hospital for their technical assistance.

No benefits in any form have been received or will be received from a commercial party related directly or indirectly to the subject of this article.

\section{References}

1. Cushing HW. Pneumatic tourniquets: with special reference to their use in craniotomies. Medical News 1904;84:577-80.

2. Klenerman L. The tourniquet in surgery. J Bone Joint Surg [Br] 1962;44-B:937-43.

3. Bruner JM. Safety factors in the use of the pneumatic tourniquet for hemostasis in surgery of the hand. J Bone Joint Surg [Am] 1951;33-A:221-4.

4. Braithwaite I, Klenerman L. Burns under tourniquets: Bruner's ten rules revisited. J Med Def Unions 1996;12:14-15.

5. Kutty S, McElwain JP. Padding under tourniquets in tourniquet controlled surgery: Bruner's ten rules revisited. Injury 2002;33:75.

6. Levy $\mathbf{0}$, David $\mathbf{Y}$, Heim M, et al. Minimal tourniquet pressure to maintain arterial closure in upper limb surgery. J Hand Surg [Br] 1993;18:204-6.

7. Van Roekel HE, Thurston AJ. Tourniquet pressure: the effect of limb circumference and systolic blood pressure. J Hand Surg [Br] 1985;10:142-4.

8. Shaw JA, Murray DG. The relationship between tourniquet pressure and underlying soft-tissue pressure in the thigh. J Bone Joint Surg [Am] 1982;64-A:1148-52.

9. BSNMedical. Velband ${ }^{\mathrm{TM}}$ Orthopaedic Bandage Rayon Bandage, 2002, http:// wound.smith-nephew.com/AU/node.asp? Node ID = 3630 (date last accessed 15th August 2006). 